A. B. Kharazishvili, Institute of Applied Mathematics, Tbilisi State

University, University str. 2, 380043 Tbilisi 43, Republic of Georgia

\title{
SOME REMARKS ON QUASIINVARIANT AND INVARIANT MEASURES
}

\begin{abstract}
We discuss two natural questions concerning some properties of quasiinvariant and invariant measures: the existence of nonmeasurable sets with respect to such measures and the existence of a nonzero $\sigma$-finite quasiinvariant measure on a proper Borel subgroup of the real line.
\end{abstract}

Let $E$ be a nonempty set, let $G$ be a group of transformations of $E$ and let $\mu$ be a $\sigma$-finite measure defined on some $\sigma$-algebra of subsets of $E$. Denote by $I(\mu)$ the family of all $\mu$-measure zero subsets of $E$. We recall that

1. $\mu$ is $G$-quasiinvariant if $\operatorname{dom}(\mu)$ and $I(\mu)$ are $G$-invariant classes of sets;

2. $\mu$ is $G$-invariant if $\operatorname{dom}(\mu)$ is a $G$-invariant class of sets and, for each $X \in \operatorname{dom}(\mu)$ and for each $g \in G$, we have $\mu(g(X))=\mu(X)$.

The most well-known example of an invariant measure is the left (right) invariant Haar measure on a locally compact topological group (see, for instance, [2]). An important special case of a Haar measure is the standard Borel measure on a finite-dimensional Euclidean space (in particular, on the real line). A Haar measure $\mu$ on a $\sigma$-compact locally compact topological group $(G, \cdot)$ has the so-called Steinhaus property: if $X \in \operatorname{dom}(\mu)$ and $\mu(X)>0$, then the set $X \cdot X^{-1}$ is a neighbourhood of the neutral element of $G$. Note that the category analogue of the Steinhaus property holds true for any topological group. Namely, if $(\Gamma, \cdot)$ is a topological group and $Y$ is a second category subset of $\Gamma$ possessing the Baire property, then the set $Y \cdot Y^{-1}$ is a neighbourhood of the neutral element of $\Gamma$.

Evidently, in order to obtain a quasiinvariant measure, it is sufficient to take an arbitrary invariant measure $\mu$ and to consider any measure equivalent to $\mu$.

Key Words: Invariant measure, quasiinvariant measure, nonmeasurable set, Haar measure

Mathematical Reviews subject classification: Primary: 28A05, 28D05

Received by the editors September 16, 1997 
However, there are some quasiinvariant probability measures which cannot be obtained in such a way. This fact implies, in particular, that some natural questions (e.g. concerning the existence of nonmeasurable sets or the existence of a measure with some additional properties) can relatively easily be solved for invariant measures, but turn out to be rather difficult for quasiinvariant measures. The main goal of this paper is to illustrate an essential difference between invariant and quasiinvariant measures from this point of view.

In our further considerations, the symbol $\mathbb{N}$ denotes the set of all natural numbers, $\mathbb{Z}$ - the set of all integers, $\mathbb{Q}$ - the set of all rational numbers and the symbol $\mathbb{R}$ denotes the real line.

We begin with one problem concerning the existence of nonmeasurable sets with respect to nonzero $\sigma$-finite quasiinvariant measures. First, let us recall that a group $G$ acts $I(\mu)$-freely in $E$ if, for any two distinct transformations $g$ and $h$ from $G$, the set $\{x \in E: g(x)=h(x)\}$ is of $\mu^{*}$-measure zero (where $\mu^{*}$ denotes, as usual, the outer measure canonically associated with $\mu$ ). The following result was obtained in [1], [3] and [9] and can be regarded as an abstract version of the classical Vitali theorem on the existence of Lebesgue nonmeasurable subsets of the real line (see [12]).

Theorem 1. Let $(E, G)$ be a space with a transformation group, let $\mu$ be a $\sigma$-finite $G$-quasiinvariant measure on $E$ and let $X$ be a subset of $E$ with $\mu^{*}(X)>0$. Suppose also that $G$ is uncountable and acts $I(\mu)$-freely in $E$. Then there exists a subset of $X$ nonmeasurable with respect to $\mu$. In particular, if $\mu$ is not identically zero, then dom $(\mu)$ differs from the family of all subsets of $E$ (in other words, $\mu$ is not an universal measure on $E$ ).

For the proof of Theorem 1, see the works mentioned above. Here we only wish to notice that the proof of this theorem is based on the classical result of Ulam [11], stating that the first uncountable cardinal $\omega_{1}$ is not real-valued measurable, and on certain properties of selectors associated with a subgroup of $G$ of cardinality $\omega_{1}$.

In connection with Theorem 1 , let us also remark that it can be essentially strengthened for $\sigma$-finite invariant measures. Namely, the following statement was recently obtained in [10].

Theorem 2. Let $(E, G)$ be a space with a transformation group, let $\mu$ be a $\sigma$-finite $G$-invariant measure on $E$ and let $X$ be a $\mu$-measurable set with $\mu(X)>0$. Suppose, in addition, that $G$ is uncountable and acts $I(\mu)$-freely in $E$. Then there exists a subset $Y$ of $X$ nonmeasurable with respect to every $G$-invariant measure on $E$ extending $\mu$ (in other words, $Y$ is absolutely nonmeasurable with respect to the class of all $G$-invariant extensions of $\mu$ ). 
Example 1. Let $E=\mathbb{R} \times \mathbb{R}$ denote the Euclidean plane, let $G$ be the group of all translations of $E$ and let $\nu$ be the classical two-dimensional Lebesgue measure on $E$. It is not difficult to show (by using the method of transfinite induction) that there exists a function $f: \mathbb{R} \rightarrow \mathbb{R}$ whose graph $Z=Z_{f}$ is a $\nu$-thick subset of $E$. In particular, we have the equality $\nu^{*}(Z)=+\infty$. At the same time, by applying the standard methods of extending invariant measures (see, for example, [6, pp. 276-287] or [4]), it can be proved that there exists a complete $G$-invariant measure $\nu^{\prime}$ on $E$ satisfying the following two conditions:

a) $\nu^{\prime}$ is an extension of $\nu$;

b) $Z \in \operatorname{dom}\left(\nu^{\prime}\right)$ and $\nu^{\prime}(Z)=0$.

We thus see that the set $Z$ (being of strictly positive outer $\nu$-measure) contains no subset absolutely nonmeasurable with respect to the class of all $G$-invariant extensions of $\nu$. Therefore, we can conclude that the assumption $X \in \operatorname{dom}(\mu)$ is rather essential in the formulation of Theorem 2. On the other hand, it is obvious that Theorem 1 does not need such an assumption.

A detailed proof of Theorem 2 is given in [10]. Unfortunately, the argument used in [10] is heavily based on the assumption of the invariance of $\mu$ and does not work for $\sigma$-finite quasiinvariant measures. Consequently, the following problem remains unsolved.

Problem 1. Let $E$ be a set, let $G$ be an uncountable group of transformations of $E$ and let $\mu$ be a $\sigma$-finite $G$-quasiinvariant measure on $E$ such that $G$ acts $I(\mu)$-freely in $E$. Can one assert that, for any $\mu$-measurable set $X$ with $\mu(X)>$ 0 , there exists a subset of $X$ absolutely nonmeasurable with respect to the class of all $G$-quasiinvariant extensions of $\mu$ ?

Moreover, this problem remains open even for the classical situation where $E$ coincides with the real line $R$ and $G$ is some uncountable group of translations of $\mathbb{R}$.

Example 2. It is well known that any Vitali subset of the real line $\mathbb{R}$ is not measurable in the Lebesgue sense and does not possess the Baire property with respect to the standard topology of $\mathbb{R}$. Furthermore, any Vitali subset of is absolutely nonmeasurable with respect to the class of all $\mathbb{Q}$-invariant measures extending the Lebesgue measure on $\mathbb{R}$. At the same time, it is not hard to demonstrate that there exist a Vitali set $X$ and a nonzero $\sigma$-finite $\mathbb{R}$-invariant measure $\mu$ on $\mathbb{R}$, for which we have $X \in \operatorname{dom}(\mu)$ (see [4, Chapter 4]). In other words, the set $X$ is not absolutely nonmeasurable with respect to the class of all nonzero $\sigma$-finite $\mathbb{R}$-invariant measures on $\mathbb{R}$ and, consequently, $X$ is not absolutely nonmeasurable with respect to the class of all $\mathbb{R}$-quasiinvariant probability measures on $\mathbb{R}$. 
In connection with Example 2, the following problem may be posed.

Problem 2. Let $(E, G)$ be a space with a transformation group and let $M$ $\left(M^{\prime}\right)$ denote the class of all nonzero $\sigma$-finite $G$-invariant ( $G$-quasiinvariant) measures on $E$. Give a characterization of all those subsets of $E$ which are absolutely nonmeasurable with respect to $M\left(M^{\prime}\right)$.

Now, we wish to discuss one question concerning the existence of invariant and quasiinvariant measures on some good (from the set-theoretical point of view) subgroups of the real line and the one-dimensional torus. First, let us consider the following simple example.

Example 3. Let $\mathbf{S}^{1}$ denote, as usual, the unit circle on the Euclidean plane, regarded as a compact topological Abelian group, and let $G$ be an uncountable Borel subgroup of $\mathbf{S}^{1}$. Suppose also that there exists a $G$-invariant Borel probability measure $\mu$ on $G$. Then one can assert that $G=\mathbf{S}^{1}$. Indeed, let us put $\mu^{\prime}(X)=\mu(X \cap G)$ for any Borel subset $X$ of $\mathbf{S}^{1}$. Clearly, $\mu^{\prime}$ is a $G$-invariant Borel probability measure on $\mathbf{S}^{1}$. Since $G$ is uncountable, there exists an element $g \in G$ of infinite order. Obviously, the set $\left\{g^{n}: n \in \mathbb{N}\right\}$ is dense in $\mathbf{S}^{1}$. Starting with this fact and taking account of the invariance of $\mu^{\prime}$ with respect to each $g^{n}(n \in \mathbb{N})$, we can easily infer that $\mu^{\prime}$ is also invariant with respect to each element of $\mathbf{S}^{1}$. Consequently, $\mu^{\prime}$ must coincide with the standard Borel probability measure on $\mathbf{S}^{1}$. Suppose for a moment that $G \neq \mathbf{S}^{1}$. Then, using the Steinhaus property of the standard Borel probability measure, we obtain that $\mu^{\prime}(G)=0$. But this is impossible since the measure $\mu^{\prime}$ is concentrated on $G$. The contradiction yields us the required equality $G=\mathbf{S}^{1}$.

A similar argument works for $\mathbb{R}$, and we easily obtain that if $G$ is an uncountable Borel subgroup of $\mathbb{R}$ for which there exists at least one $G$-invariant Borel measure $\mu$ on $G$ satisfying the relation

$$
0<\mu([0,1] \cap G)<+\infty
$$

then $G$ coincides with $\mathbb{R}$.

If we want to establish an analogous result for nonzero $\sigma$-finite quasiinvariant measures given on proper Borel subgroups of $\mathbb{R}\left(\mathbf{S}^{1}\right.$, respectively), then we need a much more difficult technique.

In order to demonstrate this, let us recall one simple notion from the general theory of topological groups (see, e.g., [2, Chapter 2]. We say that a given topological group $G$ is compactly generated if there exists a compact subset of $G$ algebraically generating $G$. 
Lemma 1. Let $G$ be a locally compact topological group and let $K$ be a compact subset of $G$. Then there exists a clopen compactly generated subgroup of $G$ containing $K$.

Proof. Denote by $e=e_{G}$ the neutral element of $G$. Clearly, the set $\{e\} \cup K$ is compact, too. Consequently, there exists an open set $U \subset G$ containing $\{e\} \cup K$ and having the compact closure. Let us put $V=\operatorname{cl}(U)$. Then it is easy to check that the group generated by $V$ is the required one.

We also need the following well-known statement which describes the structure of compactly generated locally compact Abelian groups.

Lemma 2. Let $G$ be an arbitrary compactly generated locally compact Abelian group. Then $G$ is topologically isomorphic to the product group $\mathbb{R}^{n} \times \mathbb{Z}^{m} \times G_{0}$ where $n$ and $m$ are some natural numbers and $G_{0}$ is a compact Abelian group.

Proof. The proof of this fundamental result (essentially due to Pontryagin and van Kampen) is presented in Chapter 2 of [2].

The next classical statement is due to Mackey (see [5]).

Lemma 3. Suppose that $\Gamma$ is a standard topological group (i.e. $\Gamma$ is a Borel subgroup of some Polish topological group) and suppose that $\mu$ is a left $\Gamma$-quasiinvariant Borel probability measure on $\Gamma$. Then there exists a locally compact Polish topological group $G$ with the left $G$-invariant Haar measure $\nu$, such that

1. there is an algebraic isomorphism $f: \Gamma \rightarrow G$ which simultaneously is a Borel isomorphism between the topological spaces $\Gamma$ and $G$;

2. the measure $f(\mu)$ is equivalent to the Haar measure $\nu$.

Actually, we need only relation 1 of Lemma 3 . The fact that the measures $f(\mu)$ and $\nu$ are equivalent does not play an essential role in our further considerations.

Notice that Lemma 3 with some related results is discussed in the wellknown textbook by Parthasarathy [7]. However, the presentation of this material in [7] is not quite correct.

Lemma 4. Let $\Gamma, G$ and $f$ be as in the previous lemma. Then the mapping $f^{-1}$ is a continuous bijection from $G$ onto $\Gamma$.

Proof. Evidently, $f^{-1}$ is an algebraic isomorphism between the groups $G$ and $\Gamma$. Also, $f^{-1}$ is measurable with respect to the Haar measure $\nu$ on $G$. Hence, applying the Steinhaus property of $\nu$, we obtain that $f^{-1}$ is continuous. 
Actually, the same argument yields the well-known fact that any measurable (with respect to the completion of a Haar measure) homomorphism from a $\sigma$-compact locally compact topological group into a separable topological group is continuous. Moreover, the category analogue of the Steinhaus property implies that an algebraic homomorphism from a Baire topological group into a separable topological group, having the Baire property, is necessarily continuous.

Now, we are ready to prove the following result (we do not assert that it is new, but we could not find its precise proof in the literature).

Theorem 3. Let $E$ be either $\mathbb{R}$ or $\mathbf{S}^{1}$ and let $\Gamma$ be a proper Borel subgroup of E. Suppose, in addition, that there exists a nonzero $\sigma$-finite $\Gamma$-quasiinvariant Borel measure $\mu$ on $\Gamma$. Then $\Gamma$ is at most countable.

Proof. Without loss of generality we may assume that $\mu$ is a probability measure. According to Lemma 3, there exists a locally compact Polish topological Abelian group $G$ (equipped with the Haar measure $\nu$ ) such that

1. there is an algebraic isomorphism $f: \Gamma \rightarrow G$ which simultaneously is a Borel isomorphism between the topological spaces $\Gamma$ and $G$;

2. the measure $f(\mu)$ is equivalent to the Haar measure $\nu$.

Suppose for a moment that our group $\Gamma$ is uncountable, i.e. $\operatorname{card}(\Gamma)=$ c where c denotes the cardinality of the continuum. Then we also have card $(G)=$ c. Let $K$ be an arbitrary uncountable compact subset of $G$. Applying Lemma 1, we can find a clopen compactly generated subgroup $G^{\prime}$ of $G$ containing $K$. In particular, for $G^{\prime}$, we have the equality card $\left(G^{\prime}\right)=\mathbf{c}$. Now, according to Lemma 2 , the group $G^{\prime}$ is topologically isomorphic to the product group $\mathbb{R}^{n} \times \mathbf{Z}^{m} \times G_{0}$ where $n$ and $m$ are some natural numbers and $G_{0}$ is a compact Abelian group. Further, as we know (see Lemma 4), the mapping $f^{-1}: G^{\prime} \rightarrow E$ is an injective continuous homomorphism from $G^{\prime}$ onto a proper subgroup of $E$. Consequently, $f^{-1}\left(\mathbb{R}^{n}\right)$ is a proper connected subgroup of $E$ and $f^{-1}\left(G_{0}\right)$ is a proper compact subgroup of $E$. ¿From these facts we can easily deduce that $n=0$ and that the group $G_{0}$ is finite. But this immediately implies that the group $G^{\prime}$ is at most countable, which is impossible.

The contradiction obtained finishes the proof of Theorem 3 .

Evidently, Theorem 3 remains true for any proper analytic (coanalytic) subgroup $\Gamma$ of $E$ equipped with a nonzero $\sigma$-finite $\Gamma$-quasiinvariant Borel measure $\mu$. Indeed, since $\Gamma$ is a Radon topological space, it contains a subgroup $\Gamma^{\prime}$ such that $\operatorname{card}\left(\Gamma^{\prime}\right)=\operatorname{card}(\Gamma)$ and $\Gamma^{\prime}$ is an $F_{\sigma}$-subset of $E$, and the restriction 
of $\mu$ to the Borel $\sigma$-algebra of $\Gamma^{\prime}$ is not identically equal to zero. So we may directly apply Theorem 3 to $\Gamma^{\prime}$.

However, Theorem 3 cannot be extended to the class of all projective subgroups of $E$ where $E=\mathbb{R}$ or $E=\mathbf{S}^{1}$. Indeed, in the Constructible Universe we have a projective subgroup $\Gamma$ of $\mathbb{R}$ nonmeasurable in the Lebesgue sense. For such a group $\Gamma$, the relations

$$
\Gamma \neq \mathbb{R}, \quad \lambda^{*}(\Gamma)>0, \quad \operatorname{card}(\Gamma)>\omega
$$

are fulfilled, where $\lambda$ denotes the standard Lebesgue measure on $\mathbb{R}$, and it is clear that there exists a nonzero $\sigma$-finite $\Gamma$-invariant Borel measure on $\Gamma$.

The following problem is of some interest from the methodological point of view.

Problem 3. Give a relatively elementary proof of Theorem 3 by using only the methods of real analysis and classical measure theory, which do not rely on the Mackey theorem and on the deep results concerning the structure of locally compact topological Abelian groups.

Let us remark that in [8] the author tries to present such a proof, but his argument is not quite correct.

We conclude with an example which shows that, for the Euclidean plane, the situation essentially differs from the situation of the real line.

Example 4. Let $E=\mathbb{R} \times \mathbb{R}$ and let $\Gamma=(\mathbb{R} \times\{0\})+(\{0\} \times \mathbb{Q})$. Obviously, $\Gamma$ is a proper uncountable dense $F_{\sigma}$-subgroup of $E$. We equip $\Gamma$ with the induced topology. Then it is not hard to define a nonzero $\sigma$-finite Borel measure $\mu$ on $\Gamma$ satisfying these two conditions:

a) $\mu$ is $\Gamma$-invariant;

b) $\mu$ does not have the Steinhaus property.

More precisely, condition b) says that there exists a $\mu$-measurable set $X$ with $\mu(X)>0$ for which the difference set

$$
X-X=\{x-y: x \in X, y \in X\}
$$

contains no neighbourhood of the neutral element of $\Gamma$.

\section{References}

[1] P. Erdös and R. D. Mauldin, The nonexistence of certain invariant measures, Proc. Amer. Math. Soc. 59 (1976), 321-322. 
[2] E. Hewitt and K. Ross, Abstract Harmonic Analysis, vol. 1, SpringerVerlag, Berlin, 1963.

[3] A. B. Kharazishvili, On certain types of invariant measures, Dokl. Akad. Nauk SSSR 222(3) (1975), 538-540. (in Russian)

[4] A. B. Kharazishvili, Invariant Extensions of Lebesgue Measure, Izd. Tbil. Gos. Univ., Tbilisi, 1983. (in Russian)

[5] G. W. Mackey, Borel structures in groups and their duals, Trans. Amer. Math. Soc. 85 (1957), 134-169.

[6] E. Marczewski, Collected Mathematical Papers, Polish Academy of Sciences, Institute of Mathematics, Warszawa, 1996.

[7] K. R. Parthasarathy, Introduction to Probability and Measure, Madras, 1980.

[8] D. Plachky, A note on measurable subgroups of $(\mathbf{R},+)$, preprint.

[9] C. Ryll-Nardzewski and R. Telgarsky, The nonexistence of universal invariant measures, Proc. Amer. Math. Soc. 69 (1978), 240-242.

[10] S. Solecki, On sets nonmeasurable with respect to invariant measures, Proc. Amer. Math. Soc. 119(1) (1993), 115-124.

[11] S. Ulam, Zur Masstheorie in der allgemeinen Mengenlehre, Fund. Math. 16 (1930), 140-150.

[12] G. Vitali, Sul problema della misura dei gruppi di punti di una retta, Bologna, 1905. 